Ophthalmologe 2022 $\cdot 119: 966-969$ https://doi.org/10.1007/s00347-021-01558-3 Eingegangen: 3. November 2021 Überarbeitet: 5. Dezember 2021 Angenommen: 5. Dezember 2021 Online publiziert: 3 . Januar 2022

(c) Der/die Autor(en) 2022

\section{Granulomatöse Skleritis?}

\author{
Michelle Dreesbach (D) - Thomas Reinhard · Philip Maier \\ Klinik für Augenheilkunde, Universitätsklinik Freiburg, Freiburg im Breisgau, Deutschland
}

\section{Anamnese}

Eine 54-jährige Patientin stellte sich notfallmäßig mit seit 1 Monat bestehender lokalisierter Rötung temporal am rechten Auge sowie deutlichem Fremdkörpergefühl in der Klinik für Augenklinik, Universitätsklinik Freiburg vor. Außer einer Hyperopie, einer Schieloperation rechts (1972) und einer bekannten Frühblüherallergie war die ophthalmologische Anamnese unauffällig. Vom externen Kollegen wurde die Patientin mit Ofloxacin-Augentropfen (URSAPHARM, Saarbrücken, Deutschland) (5-mal täglich) 1 Woche lang und anschließend mit Dexamethason-Augentropfen für 3 Tage (Dr. Gerhard Mann chem.pharm. Fabrik GmbH, Berlin, Deutschland) (1-mal täglich) vorbehandelt. Zum Zeitpunkt der Untersuchung zeigten sich keine Allgemeinsymptome und keine Infektkonstellation. Die Patientin gab an, keine systemischen Medikamente einzunehmen.

\section{Klinischer Befund}

Der Visus betrug bei der Erstvorstellung 1,0 beidseits, der Augeninnendruck betrug $19 \mathrm{~mm} \mathrm{Hg}$ rechts und $15 \mathrm{~mm} \mathrm{Hg}$ links. Am rechten Auge zeigte sich spaltlampenbiomikroskopisch eine temporal lokalisierte Rötung der Bindehaut mit einem gelblich durchscheinenden erhabenen Tumor (- Abb. 1). Die sonstigen Befunde des vorderen Augenabschnittes waren beidseits unauffällig. Fundoskopisch war am rechten Auge an der temporalen Netzhaut eine sehr flache seröse Abhebung zu sehen. Ansonsten zeigte sich ein funduskopischer Normalbefund. Die diskrete seröse Netzhautabhebung ließ sich mittels Ultraschallbiomikroskopie bestätigen. Zudem zeigte sich in der Hochfrequenzsonographie (Gerätehersteller: AVISO S, Quantel medical, Cournon d'Auvergne, Frankreich, $50 \mathrm{MHz}$ Ultraschallbiomikroskopie) an der Stelle der lokalisierten Rötung eine Prominenz der Sklera mit hypo- und hyperreflektivem Material (- Abb. 2a, b).

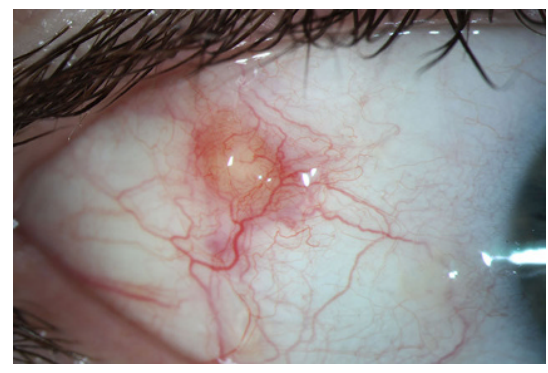

Abb. 1 \ Spaltlampenbiomikroskopische Aufnahmen des Primärbefundes: Es zeigt sich eine umschriebene konjunktivale Rötung mit einer nodulären granulomatösen Veränderung (Foto vom März 2020) 

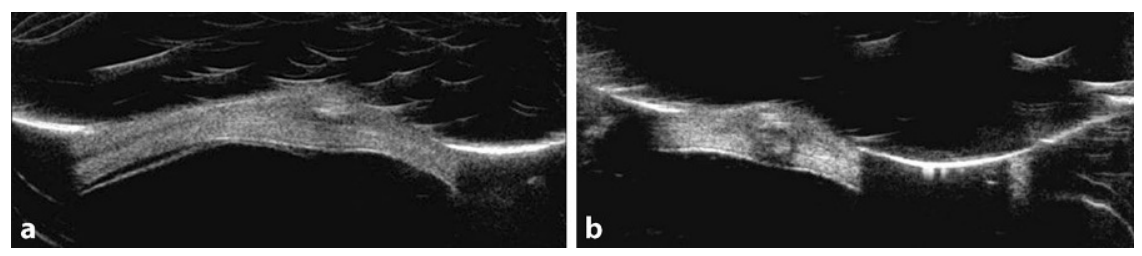

Abb. 2 \ Hochfrequenzsonographie (Gerätehersteller: AVISOS, Quantel medical,50 MHz Ultraschallbiomikroskopie). a Es zeigt sich temporal eine aufgelockerte Sklera mit sehr flacher leicht seröser Netzhautablösung. b An der Stelle der nodulären Erhabenheit zeigt sich eine dichte Struktur mit sowohl hypo- und hyperreflektivem Material, welche eine Größe von $2 \times 1,7 \times 1,4 \mathrm{~mm}$ misst. Zudem zeigt sich unter dem hyperreflektiven Material ein deutlicher Schallschatten

\section{Wie lautet Ihre Diagnose?}

\section{Procedere}

In Zusammenschau der Befunde (lokalisierte Rötung, kleine seröse Amotio) vermuteten wir am ehesten eine noduläre Skleritis mit seröser Amotio am rechten Auge. Unter einer Therapie mit Dexamethason-Augentropfen (insgesamt über 17 Tage mit Tropfreduktion) und anschlieBend mit Nevanac 0,3\%-Augentropfen (S.A. Alcon-Couvreur N.V., Puurs, Belgien) 1-mal täglich und Voltaren $150 \mathrm{mg}$ (Novartis Pharma GmbH, Wien, Österreich) systemisch zeigte sich 6 Wochen später zwar nur eine geringe Besserung der subjektiven Beschwerden, jedoch ein deutlicher Rückgang der konjunktivalen Rötung (『Abb. 3). Bei verbesserter Bindehauttransparenz bestand nun der Verdacht auf intrasklerale Fadenreste nach der ehemaligen Schieloperation. Leider besaß die Patientin keinerlei Vorbefunde oder Operationsberichte bezüglich der Schieloperation 1972.

Wir entscheiden uns daher für eine Ausschneidung und Biopsie des subkonjunktivalen Materials ggf. mit Defektdeckung.
Zudem sollten vorsorglich die 3 weiteren erkennbaren subkonjunktivalen Fadenreste entfernt werden. Während der Operation wurde die Bindehaut eröffnet, und es zeigte sich ein tief liegender Faden mit umgebender Entzündungsreaktion temporal. Der nahezu intakte Faden sowie das umgebende entzündliche Gewebe konnten vollständig entfernt und die Bindehaut anschließend mit resorbierbarem Fadenmaterial wieder adaptiert werden.

\section{Diagnose und Verlauf}

Es wurde letztlich die Diagnose einer fremdkörperinduzierten Skleritis mit Fremdkörpergranulom nach Schieloperation 1972 gestellt.

Postoperativ zeigte sich eine gut adaptierte Bindehaut ohne Wunddehiszenz oder weitere Inflammation der Sklera. Wir leiteten eine Lokaltherapie mit PrednisoIon-Augentropfen 5-mal (Allergan $\mathrm{GmbH}$, Wiesbaden, Deutschland) täglich ein, welche nach einem Ausschleichschema reduziert wurde. Drei Wochen später zeigte sich ein stabiler Befund ohne Hinweis auf eine erneute Entzündungsreaktion (• Abb.4), die dezente seröse Netzhautablösung war noch nicht vollständig resorbiert. Die Patientin war beschwerdefrei, sodass bei stabilem Netzhaut- und Vorderabschnittsbefund keine weitere Therapie indiziert erschien und jährliche Verlaufskontrollen empfohlen wurden.

\section{Diskussion}

Subkonjunktivale Granulome wie in dem hier geschilderten Fall stellen eine sehr seltene Komplikation nach einer Schieloperation dar. Sie sind die Antwort auf eine Verletzung der Bindehaut bzw. der Sklera meist nach operativen Eingriffen oder eine Reaktion auf traumatisch eingebrachtes Fremdkörpermaterial. Sie entstehen in der Regel im Rahmen einer verzögerten Wundheilung durch eine vermehrte vasoproliferative inflammatorische Antwort bei der Wundgranulation und treten meist innerhalb von Wochen bis Monaten nach einem operativen Eingriff auf. Das Auftreten eines solchen Befundes mehrere Jahr-

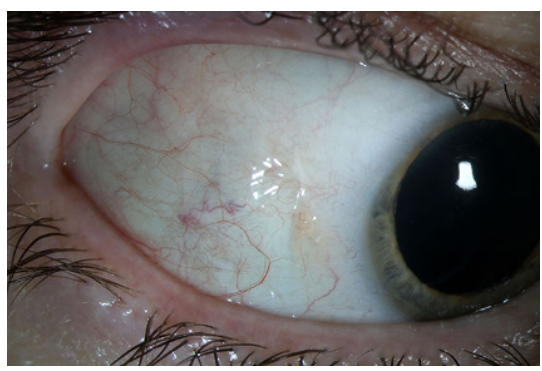

Abb. $3 \Delta$ a Spaltlampenbiomikroskopische Aufnahmen: 8 Wochen nach topischer und systemischer antiinflammatorischer Behandlung zeigt sich ein leichter Rückgang der Injektion bei weiterhin bestehender deutlicher Prominenz der temporalen Bindehaut. b Intraoperativ zeigt sich ein vollständig intakter Faden als Verursacher des Fremdkörpergranuloms fast 50 Jahre nach der Schieloperation
Abb. 4 \ Spaltlampenbiomikroskopische Aufnahme: Reizfreier Befund 3 Monate nach Exzision des Fremdkörpermaterials. Subjektiv besteht Beschwerdefreiheit

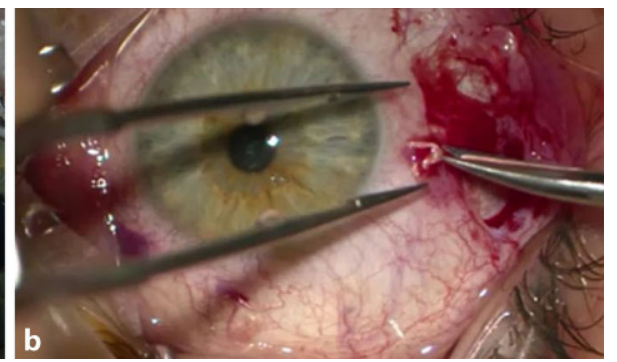


zehnte nach dem Eingriff wie im hier geschilderten Fall ist sehr ungewöhnlich [5].

Differenzialdiagnostisch war in erster Linie an eine noduläre Skleritis zu denken. Ohne eine zeitlich assoziierte Traumaanamnese erschien eine epitheliale Einschlusszyste oder ein pyogenes Granulom weniger wahrscheinlich.

Epitheliale Einschlusszysten können durch die Einwanderung von Epithelzellen in die Substantia propria mit anschließender Proliferation und Bildung eines zystischen Hohlraumes entstehen. Jede Operation mit Verletzung der Bindehaut kann Ursache für die Entstehung einer solchen Zyste sein. Pyogene Granulome stellen häufige benigne vaskuläre Bindehauttumoren dar, welche oft gestielt sind, schnell an Größe zunehmen und eine hohe Blutungsneigung haben. Sie sind meist selbst limitierend und reagieren wenig auf eine Lokaltherapie [4]. Zur Diagnosesicherung wird oftmals eine Exzision durchgeführt. Dabei fanden Al-Towerki et al., dass von 110 beobachteten Fällen $80 \%$ der pyogenen Granulome nach einer vorangegangenen Operation auftraten [1].

In unserem Fall zeigten sich keine gestielte Läsion der Bindehaut mit erhöhter Blutungsneigung und zudem keine zystische Raumforderung, welche diese Differenzialdiagnosen insbesondere beim Fehlen einer zeitlich assoziierten Operation oder eines Traumas unwahrscheinlich machten.

In der Literatur sind nur wenige Einzelfälle von Granulombildungen in Assoziation mit nichtresorbierbarem Fadenmaterial beschrieben, hier sind sowohl pyogene $[2,3,6]$ als auch Faden-assoziierte Fremdkörpergranulome beschrieben [7]. Im Jahr 2005 beschrieben Espinoza et al. [2] das Auftreten von pyogenen Granulomen nach einer Schieloperation in 2,1\% (10 von 472 Patienten), wohingegen bei Makino et al. [6] eine Inzidenz von 0,39\% (2 von 512 Patienten) gefunden wurde. Dabei trat die granulomatöse Entzündungsreaktion stets im zeitlichen Abstand von ca. 1 Monat zur Schieloperation auf. In der Studie von Espinoza et al. [2] wurde die Bindehaut nach der Schieloperation mittels Kauter verschlossen, bei der Studie von Makino et al. [6] wurden wie in unserem Fall nicht resorbierbare Fäden für die Muskelfixation verwen- det. Als Ursache der Entzündungsreaktion wurde hier ein nichtadäquater Wundverschluss der Bindehaut mit offen liegender Sklera vermutet. Mathew et al. [7] berichteten von einem 10 Jahre alten Mädchen, welches 15 Monate nach der Schieloperation mit nichtresorbierbaren Fäden für die episklerale Fixation der Augenmuskeln ein Granulom nahe der Plica entwickelte. Es zeigte sich unter lokaler Kortisontherapie kein Rückgang der Schwellung. Bei fehlenden Beschwerden und Ablehnung eines operativen Eingriffs wurde das Granulom belassen. Als Ursache der Entzündungsreaktion wurde vermutet, dass das Fadenende nicht ausreichend von Bindehaut bedeckt wurde.

\section{\) Diagnose: Subkonjunktivales Granulom durch Fadenreste nach einer Schieloperation in der Kindheit}

Unser Fall zeigt, dass sich auch viele Jahre nach einer Schieloperation mit nicht resorbierbarem Fadenmaterial ein subkonjunktivales intrasklerales Fremdkörpergranulom mit begleitender Skleritis ausbilden kann. Therapeutisch brachte letztendlich nur die operative Exzision des Fremdkörpers eine dauerhafte Symptombesserung. Denn zuvor zeigte sich nur eine geringe Besserung unter lokaler und systemischer antiphlogistischer Therapie. Weshalb es in unserem Fall mehrere Jahrzehnte nach der Schieloperation zu einer derartigen Fremdkörperreaktion gegen das Fadenmaterial kam, bleibt unbeantwortet.

\section{Fazit für die Praxis}

Fremdkörperinduzierte Granulome können an den Augen nach Verletzungen oder auch nach operativer Manipulation auftreten. Sie treten meist innerhalb von Wochen bis Monaten nach einer Operation oder einem Trauma auf, können jedoch auch, wie unser Fall zeigt, viele Jahre nach einem operativen Eingriff auftreten und sollten differenzialdiagnostisch berücksichtigt werden.

Bei dem Befund einer nodulären Entzündungsreaktion unklarer Ursache und bei Nichtansprechen auf eine topische und systemische antiphlogistische Therapie sollte bei Verdacht (z. B. bei einer anamnestisch zurückliegenden Operation oder einem Trauma) immer mittels einer Exzision Fremdkörpermaterial ausgeschlossen und bei Vorhandensein entfernt werden. Des Weiteren kann die (exzisionale) Biopsie in derartigen Situationen auch helfen, weitere differenzialdiagnostische Ursachen wie eine akute nekrotisierende Skleritis und in seltenen Fällen auch ein malignes Geschehen auszuschließen.

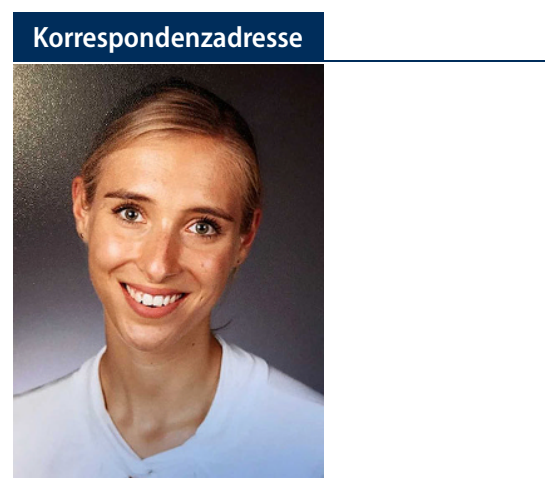

Dr. Michelle Dreesbach

Klinik für Augenheilkunde, Universitätsklinik Freiburg

Kilianstr. 5, 79106 Freiburg im Breisgau,

Deutschland

michelle.dreesbach@uniklinik-freiburg.de

Funding. Open Access funding enabled and organized by Projekt DEAL.

\section{Einhaltung ethischer Richtlinien}

Interessenkonflikt. M. Dreesbach, T. Reinhard und P. Maier geben an, dass kein Interessenkonflikt besteht.

Für diesen Beitrag wurden von den Autoren keine Studien an Menschen oder Tieren durchgeführt. Für die aufgeführten Studien gelten die jeweils dort angegebenen ethischen Richtlinien. Für Bildmaterial oder anderweitige Angaben innerhalb des Manuskripts, über die Patienten zu identifizieren sind, liegt von ihnen und/oder ihren gesetzlichen Vertretern eine schriftliche Einwilligung vor.

Open Access. Dieser Artikel wird unter der Creative Commons Namensnennung 4.0 International Lizenz veröffentlicht, welche die Nutzung, Vervielfältigung, Bearbeitung, Verbreitung und Wiedergabe in jeglichem Medium und Format erlaubt, sofern Sie den/die ursprünglichen Autor(en) und die Quelle ordnungsgemäß nennen, einen Link zur Creative Commons Lizenz beifügen und angeben, ob Änderungen vorgenommen wurden.

Die in diesem Artikel enthaltenen Bilder und sonstiges Drittmaterial unterliegen ebenfalls der genannten Creative Commons Lizenz, sofern sich aus der Abbildungslegende nichts anderes ergibt. Sofern das betreffende Material nicht unter der genannten Creative Commons Lizenz steht und die betreffende Handlung nicht nach gesetzlichen Vorschriften erlaubt ist, ist für die oben aufgeführten Weiterverwendungen des Materials die Einwilligung des jeweiligen Rechteinhabers einzuholen. 


\section{Literatur}

1. Al-Towerki AA (1996) Pyogenic granuloma. Int Ophthalmol 19(5):287-291

2. Espinoza GM, Lueder GT (2005) Conjunctival pyogenic granulomas after strabismus surgery. Ophthalmology 112(7):1283-1286

3. Gal A, France TD (1986) Granuloma formation as a complication of the posterior fixation suture operation. Arch Ophthalmol 104:1755

4. Herwig-Carl MC, Grossniklaus $\mathrm{HE}$, Müller PL, Atzrodt L, Loeffler KU, Auw-Haedrich C (2019) Pyogenic granuloma associated with conjunctival epithelial neoplasia: report of nine cases. $\mathrm{Br} J$ Ophthalmol 103(10):1469-1474

5. Jordan DR, Brownstein S, Lee-Wing M, Ashenhurst $M$ (2001) Pyogenic granuloma following oculoplastic procedures: an imbalance in angiogenesis regulation? Can J Ophthalmol 36(5):260-268

6. Makino S, Hozawa K (2014) Conjunctival pyogenic granuloma after strabismus surgery. Imaging J Clin Med Sciences 1(2):13

7. Mathew AA, Kowal L (2017) Conjunctival granuloma post pulley fixation suture. J AAPOS 21(4):342-343

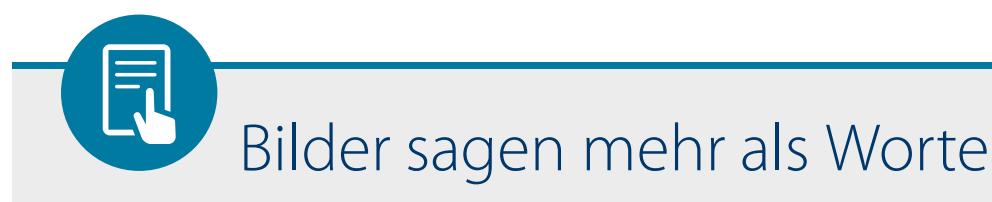

Wir suchen Ihre informativen und überraschenden Bilder!

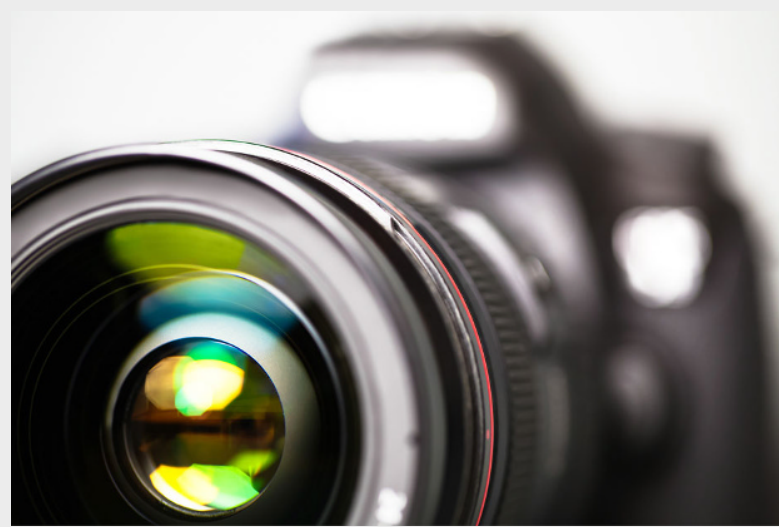

(c) Fotimmz / Fotolia sche Korrelation mit einer prägnanten und aussagekräftigen Bildlegende.

Eine Auswahl der informativsten Schnappschüsse und klinisch-pathologischen Korrelationen werden dann, inklusive der Geschichte dahinter, in Die Ophthalmologie veröffentlicht. Wir freuen uns auf Ihre Beteiligung!

\section{Hinweise zur Einreichung:}

- 1 Abbildung bestehend aus max. sechs Einzelbildern (a-f); bei klinisch-pathologischer Korrelation unter Angabe von Färbung und Vergrößerung

- Aussagekräftiger Manuskript-Titel

- Bildlegende mit max. 2500 Zeichen inkl. Leerzeichen

- Max. 4 Autorinnen/Autoren sowie vollständige Korrespondenzadresse

Senden Sie Ihre Bilder an:

Michal Meyer zu Tittingdorf Managing Editor von Die Ophthalmologie michal.meyerzutittingdorf@springer.com die aufschlussreichsten Bilder aus Ihrem Alltag mit der Community zu teilen.

Schicken Sie uns Ihre Aufnahme oder eine klinisch-pathologi- 